\title{
Correction to: Ten-year results of accelerated hypofractionated adjuvant whole-breast radiation with concomitant boost to the lumpectomy cavity after conserving surgery for early breast cancer
}

\author{
Domenico Cante $^{1}$ - Edoardo Petrucci ${ }^{2}$. Piera Sciacero ${ }^{1}$. Cristina Piva ${ }^{1} \cdot$ Silvia Ferrario ${ }^{1}$ - Silvia Bagnera ${ }^{3}$. \\ Sebastiano Patania ${ }^{3}$. Guido Mondini ${ }^{4} \cdot$ Massimo Pasquino $^{2} \cdot$ Valeria Casanova Borca $^{2} \cdot$ Giorgio Vellani $^{5}$. \\ Maria Rosa La Porta ${ }^{1} \cdot$ Pierfrancesco Franco ${ }^{6}$ (i)
}

Published online: 12 December 2017

(c) Springer Science+Business Media, LLC, part of Springer Nature 2017

\section{Correction to: Med Oncol (2017) 34:152 \\ https://doi.org/10.1007/s12032-017-1020-4}

The original version of this article unfortunately contained a mistake. The citation of local relapse rates of the EORTC
22881-10882 trial ('boost vs no boost trial') was incorrect. The corrected sentence is given below

"The EORTC "boost vs no boost" trial demonstrated a 10 -year cumulative incidence of local relapse of $6.2 \%$ for the boost arm compared to $10.2 \%$ for the no boost arm"

The original article can be found online at https://doi.org/10.1007/s12032-017-1020-4.

Pierfrancesco Franco

pierfrancesco.franco@unito.it

1 Radiotherapy Department, Ivrea Community Hospital, ASL TO4, Ivrea, Italy

2 Medical Physics Department, Ivrea Community Hospital, ASL TO4, Ivrea, Italy

3 Diagnostic Imaging Department, Ivrea Community Hospital, ASL TO4, Ivrea, Italy

4 Breast Surgery Department, Ivrea Community Hospital, ASL TO4, Ivrea, Italy

5 Medical Oncology Department, Ivrea Community Hospital, ASL TO4, Ivrea, Italy

6 Department of Oncology - Radiation Oncology, University of Turin, Via Genova 3, 10126 Turin, Italy 